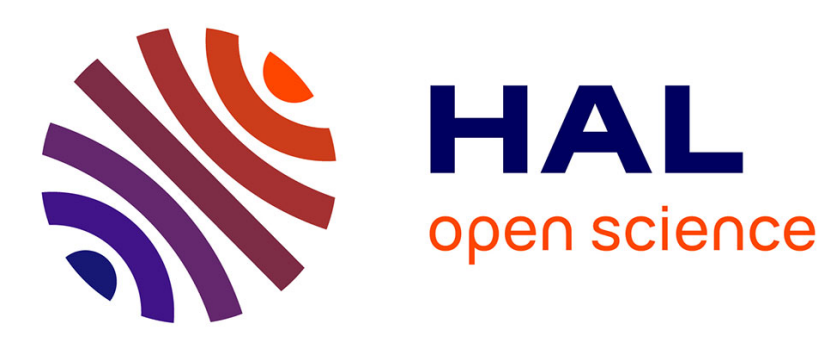

\title{
HDR Image Tone Mapping Approach based on Near Optimal Separable Adaptive Lifting Scheme
}

Ba Chien Thai, Anissa Mokraoui, Basarab Matei

\section{To cite this version:}

Ba Chien Thai, Anissa Mokraoui, Basarab Matei. HDR Image Tone Mapping Approach based on Near Optimal Separable Adaptive Lifting Scheme. International Conference on Signal Processing: Algorithms, Architectures, Arrangements, and Applications, Sep 2018, Poznan, Poland. 10.23919/SPA.2018.8563293 . hal-02428256

\section{HAL Id: hal-02428256 \\ https://hal.science/hal-02428256}

Submitted on 5 Jan 2020

HAL is a multi-disciplinary open access archive for the deposit and dissemination of scientific research documents, whether they are published or not. The documents may come from teaching and research institutions in France or abroad, or from public or private research centers.
L'archive ouverte pluridisciplinaire HAL, est destinée au dépôt et à la diffusion de documents scientifiques de niveau recherche, publiés ou non, émanant des établissements d'enseignement et de recherche français ou étrangers, des laboratoires publics ou privés. 


\title{
HDR Image Tone Mapping Approach based on Near Optimal Separable Adaptive Lifting Scheme
}

\author{
Ba chien Thai*, Anissa Mokraoui* and Basarab Matei ${ }^{\dagger}$ \\ ${ }^{*}$ L2TI, ${ }^{\dagger}$ LIPN, Institut Galilée, Université Paris 13 Sorbonne Paris Cité \\ 99, Avenue Jean-Baptiste Clément, 93430 Villetaneuse, France \\ \{bachien.thai, anissa.mokraoui\}@univ-paris13.fr \\ matei@lipn.univ-paris13.fr
}

\begin{abstract}
This paper proposes a Tone Mapping (TM) approach converting a High Dynamic Range (HDR) image into a Low Dynamic Range (LDR) image while preserving as much information of the HDR image as possible to ensure a good LDR image visual quality. This approach is based on a separable near optimal lifting scheme using an adaptive powerful prediction step. The latter relies on a linear weighted combination depending on the neighboring coefficients extracting then the relevant finest details in the HDR image at each resolution level. Moreover the approximation and detail coefficients are modified according to the entropy of each subband. The pixel's distribution of the coarse reconstructed LDR image is then adjusted according to a perceptual quantizer with respect to the human visual system using a piecewise linear function. Simulation results provide good results, both in terms of visual quality and TMQI metric, compared to existing competitive TM approaches.
\end{abstract}

\section{INTRODUCTION}

The objective of a High Dynamic Range (HDR) image Tone Mapping (TM) approach is to find a trade-off between the relevant information (e.g. details, contrast, brightness...) to be preserved or discarded in the image ensuring a good visual quality of the displayed image on Low Dynamic Range (LDR) devices that would be appreciated by observers.

A state of the art on HDR image TM approaches is fairly complete in [1], [2] and [3] where a classification is provided. Among the developed TM strategies, this paper quickly reviews those that caught our attention because of their performance. In [6], the TM approach reduces the HDR contrast while preserving the image details. This work uses an edge-preserving bilateral filter to decompose the HDR image into two layers: a base layer encoding large-scale variations and a detail one. Contrast is then reduced only in the first layer while the details are kept unchanged. An adaptive logarithmic mapping method of luminance values is presented in [7]. It concerns the adjustment of the logarithmic basis depending on the radiance of the pixels. In [8], a subband architecture related on an oversampled Haar pyramid representation is proposed. Subband coefficients are re-scaled according to a gain control function reducing the high frequency magnitudes and boosting low ones. In [9], a TM optimization approach using a histogram adjustment between linear mapping and the equalized histogram mapping is developed. A modification of this approach is made where revisited histogram equalization approaches are discussed in [10]. The latter considers both histogram equalization and human sensitivity to the light function.

In [11], a second generation of wavelets based on the edge content of the image avoiding having pixels from both sides of an edge is proposed. In [12], a separable non-linear multiresolution approach based on essentially non-oscillatory interpolation strategy has been investigated. These approaches take into account the singularities (e.g. edge points) in their mathematical models preserving then the structural information of the HDR images. In [13], a non-separable non-linear multiresolution approach is proposed. The results provided in [11], [12] and [13] show that the decomposition of the HDR image on different resolution levels would seem to be a good strategy. However, the choice of the decomposition filters is extremely important and decisive in the extractive power of the approximation and detail information. To do so, this paper proposes a separable near optimal lifting scheme. The "Predict" operation is performed by a new adaptive prediction operation to extract the finest detail coefficients to highlight the sharp transitions in the given HDR image.

The proposed HDR image TM approach has two main goals namely the preservation of the details and the adjustment of the contrast in accordance with the LDR display devices. It is composed of four stages. The first one judiciously decomposes the HDR image into different resolution levels (section II-A). The second one concerns the weighting strategy of the detail and approximation coefficients (section II-B). The third one reconstructs the coarse LDR image (section II-C). Finally the fourth one adjusts the contrast according to a perceptual linear quantizer (section II-D). Section III discusses the simulation results. Section IV concludes the paper.

\section{Proposed HDR IMAGE TONE MAPPING APPROACH}

This section concerns the first, second, third and fourth stages of the proposed HDR image TM mapping approach (i.e. decomposition, weighting, reconstruction and contrast adjustment stages).

Before describing the approach, introduce some notations. The original HDR image, at the finest resolution level $J$, is assumed to be of size $N^{J} \times M^{J}$. The index $j$ refers to the resolution level (with $j=0, \ldots, J-1$ ). Denote $l_{H D R}$ the HDR image luminance. In the rest of this paper, the HDR image luminance is considered in the logarithm domain since it is 
well adapted to the human visual system. It is denoted $I^{J}$ and defined as follows $I^{J}=\left\{I^{J}\left(x_{n}, y_{m}\right)=\log _{10}\left(l_{H D R}\left(x_{n}, y_{m}\right)\right)\right.$ for $1 \leq n \leq N^{J}$ and $1 \leq m \leq M^{J}$ where $I^{J}\left(x_{n}, y_{m}\right)$ is the HDR logarithm luminance value of the pixel located at position $\left(x_{n}, y_{m}\right)$ on the image.

\section{A. First stage: HDR image decomposition}

The proposed HDR image decomposition is performed according to the forward process of a separable near optimal cell-average lifting scheme. This strategy is motivated by the fact that the relevant details are accurately predicted since the coefficients of the filters are adapted locally to the image to be processed.

The decomposition consists to go from the finest resolution level $J$ to the coarse resolution level 0 . At a given resolution level $j-1$ (with $0 \leq j \leq J-1$ ), the algorithm deals with the approximation coefficients denoted $I^{j}\left(x_{n}, y_{k}\right)$ (with $1 \leq n \leq$ $N^{j}$ and $1 \leq k \leq M^{j}$ ) computed at resolution level $j$. For a given $n$ belonging to $\left[1, N^{j}\right]$, the algorithm starts with splitting the horizontal 1D-signal, i.e. $I^{j}\left(x_{n}, y_{k}\right)$ for $1 \leq k \leq M^{j}$, into a set of odd and even indexes as follows:

$$
\begin{array}{r}
\left\{I^{j}\left(x_{n}, y_{k}\right) \text { with } 1 \leq k \leq M^{j}\right\} \\
:=\left\{I^{j}\left(x_{n}, y_{2 k-1}\right), I^{j}\left(x_{n}, y_{2 k}\right) \text { with } 1 \leq k \leq M^{j} / 2\right\} .
\end{array}
$$

This process is then repeated for all $n$. Based on this split, the approximation coefficient located at position $\left(x_{n}, y_{k}\right)$, denoted $V^{j-1}\left(x_{n}, y_{k}\right)$, is computed on a Cell-Average (CA) scheme. For a given $n$, it is expressed as follows:

$$
\begin{array}{r}
V^{j-1}\left(x_{n}, y_{k}\right)=\frac{I^{j}\left(x_{n}, y_{2 k-1}\right)+I^{j}\left(x_{n}, y_{2 k}\right)}{2} \\
\text { for } 1 \leq k \leq M^{j} / 2 .
\end{array}
$$

This process is then repeated for all $n$.

The detail coefficient, denoted $d^{j-1}\left(x_{n}, y_{2 k-1}\right)$, is computed at odd indexes and is provided below for a given $n$ :

$$
\begin{array}{r}
d^{j-1}\left(x_{n}, y_{2 k-1}\right)=\hat{I}^{j}\left(x_{n}, y_{2 k-1}\right)-I^{j}\left(x_{n}, y_{2 k-1}\right) \\
\text { for } 1 \leq k \leq M^{j} / 2,
\end{array}
$$

where $\hat{I}^{j}\left(x_{n}, y_{2 k-1}\right)$ is the predicted logarithm luminance value at odd position $\left(x_{n}, y_{2 k-1}\right)$ and resolution level $j$. This predicted value is expressed as a linear weighted combination of the neighboring approximation coefficients at resolution level $j-1$ :

$$
\begin{array}{r}
\hat{I}^{j}\left(x_{n}, y_{2 k-1}\right)=\sum_{i=0}^{2} u_{i}^{j-1}\left(x_{n}\right) \cdot V^{j-1}\left(x_{n}, y_{k+i-1}\right) \\
\text { for } 1 \leq k \leq M^{j} / 2,
\end{array}
$$

where the weights $u_{i}^{j-1}\left(x_{n}\right)$ must preserve the initial 1Dsignal average which results in satisfying this condition:

$$
\sum_{i=0}^{2} u_{i}^{j-1}\left(x_{n}\right)=1 .
$$

In what follows, the weights are rather denoted in a vector form $\mathbf{u}^{j-1}=\left(u_{0}^{j-1}\left(x_{n}\right), u_{1}^{j-1}\left(x_{n}\right), u_{2}^{j-1}\left(x_{n}\right)\right)^{t}$ to lighten the writing. These weights are deduced so that the Mean Squared
Error (MSE) between $\hat{I}^{j}\left(x_{n}, y_{2 k-1}\right)$ and $I^{j}\left(x_{n}, y_{2 k-1}\right)$ is minimized:

$$
\begin{array}{r}
E=\underset{u_{i}^{j-1}}{\arg \min }\left\|\hat{I}^{j}\left(x_{n}, y_{2 k-1}\right)-I^{j}\left(x_{n}, y_{2 k-1}\right)\right\|_{2}^{2} \\
\text { for } 1 \leq k \leq M^{j} / 2 .
\end{array}
$$

This results in solving the following equation:

$$
\Gamma^{j-1} \cdot \mathbf{u}^{j-1}=\mathbf{r}^{j-1}
$$

where $\mathbf{u}^{j-1}$ is the weight vector. $\mathbf{r}^{j-1}$ is the crosscorrelation vector $\mathbf{r}^{j-1}=\left(r^{j-1}(0), r^{j-1}(1), r^{j-1}(2)\right)^{t}$ where $r^{j-1}(i)$ represents the cross-correlation function between $V^{j-1}\left(x_{n}, y_{k+i-1}\right)$ and $I^{j}\left(x_{n}, y_{2 k-1}\right)$ for $1 \leq k \leq M^{j} / 2$. $\Gamma^{j-1}$ is the autocorrelation matrix defined as:

$$
\boldsymbol{\Gamma}^{j-1}=\left[\begin{array}{lll}
R^{j-1}(0) & R^{j-1}(1) & R^{j-1}(2) \\
R^{j-1}(-1) & R^{j-1}(0) & R^{j-1}(1) \\
R^{j-1}(-2) & R^{j-1}(-1) & R^{j-1}(0)
\end{array}\right],
$$

where $R^{j-1}(i)$ is the autocorrelation of $V^{j-1}\left(x_{n}, y_{k}\right)$.

These weights, associated to the row $\left(x_{n}\right)$, are then deduced so that the partial derivatives of the MSE (given by equation (6)) with respect to $u_{i}^{j-1}$ (i.e. $i=0,1,2$ ) are equal to zero:

$$
\mathbf{u}^{j-1}\left(x_{n}\right)=\left(\boldsymbol{\Gamma}^{j-1}\right)^{-1} \cdot \mathbf{r}^{j-1} \text {. }
$$

The weight vectors are then stored to be used in the adaptive lifting scheme backward process to reconstruct the decomposed 1D-signal.

For sake of convenience, the approximation and details coefficients are organized as follows:

$$
\begin{array}{r}
\quad\left\{W^{j}\left(x_{k}, y_{m}\right) \text { for } 1 \leq k \leq N^{j} \text { and } \forall m \in\left[1, M^{j} / 2\right]\right\} \\
:=\left\{V^{j-1}\left(x_{n}, y_{k}\right) \text { for } 1 \leq k \leq M^{j} / 2 \text { and } \forall n \in\left[1, N^{j}\right]\right\}, \\
\left\{U^{j}\left(x_{k}, y_{m}\right) \text { for } 1 \leq k \leq N^{j}, \forall m \in\left[1, M^{j} / 2\right]\right\} \\
:=\left\{d^{j-1}\left(x_{n}, y_{2 k-1}\right) \text { for } 1 \leq k \leq M^{j} / 2, \forall n \in\left[1, N^{j}\right]\right\} .
\end{array}
$$

The split, approximation and detail operations are applied on $W^{j}\left(x_{k}, y_{m}\right)$ and $U^{j}\left(x_{k}, y_{m}\right)$ for a given $m \in\left[1, M^{j} / 2\right]$ (i.e. on the vertical direction). Note that the approximation step requires the prediction of $\hat{W}^{j}\left(x_{2 k-1}, y_{m}\right)$ (respectively $\hat{U}^{j}\left(x_{2 k-1}, y_{m}\right)$ ) based on a set of weights $\mathbf{v}_{i}^{j-1}$ (respectively $\mathbf{w}_{i}^{j-1}$ ). These weights need to be stored for the backward process lifting scheme to reconstruct the decomposed image. Finally, the approximation resolution level $I^{j}\left(x_{n}, y_{m}\right)$ is divided into 4 blocks $I^{j}:=\left(I^{j-1}, d_{H L}^{j-1}, d_{L H}^{j-1}, d_{H H}^{j-1}\right)$. The decomposition is thus iterated on $I^{j-1}$ until $j=0$.

The finest HDR image $I^{J}$ is then represented by $3 J+1$ resolution levels $I^{J}:=$ $\left(I^{0}, d_{H L}^{0}, d_{L H}^{0}, d_{H H}^{0}, \ldots, d_{H L}^{J-1}, d_{L H}^{J-1}, d_{H H}^{J-1}\right)$.

\section{B. Second stage: Weighting strategy of the coefficients}

To reduce the dynamic range of the HDR image, the TM approach proposes to weight the approximation and detail coefficients in an appropriate way before performing the adaptive lifting scheme backward process described in section II-C. 
Denote $N_{l}$ the number of resolution levels equal to $J ; E_{a}$ the entropy of the approximation coefficients at the coarsest resolution level (i.e. $j=0$ ); and $E_{d}^{j}$ the entropy of the detail coefficients at resolution level $j$ (i.e. $d_{H L}^{j}, d_{L H}^{j}$ and $d_{H H}^{j}$ ) getting therefore $N_{l}+1$ entropies $\left(E_{a}, E_{d}^{0}, E_{d}^{1}, \ldots, E_{d}^{j}, \ldots\right.$, $\left.E_{d}^{N_{l}-1}\right)$. From these entropies, positive weights smaller than one are deduced as follows:

$$
\left\{\begin{array}{l}
\alpha_{a}=\frac{\sum_{i=0}^{N_{l}-1} E_{d}^{i}}{E_{a}+\sum_{i=1}^{N_{l}-1} E_{d}^{i}} \text { for } j=0 \\
\alpha_{d}^{j}=\frac{E_{a}+\sum_{i=0, \neq j}^{N_{l}-1} E_{d}^{i}}{E_{a}+\sum_{i=0}^{N_{l}-1} E_{d}^{i}} \text { for } j=0, \ldots, N_{l}-1
\end{array}\right.
$$

$\alpha_{a}$ (respectively $\alpha_{d}^{j}$ ) is the weight associated to the approximation (respectively detail) coefficients at resolution level $j=0$ (respectively $j$ ).

The coefficients of the four coarsest resolution levels are first modified according to:

$$
\begin{aligned}
I^{\prime 0}=\alpha_{a} \times I^{0}, & d_{H L}^{\prime 0}=\alpha_{d}^{0} \times d_{H L}^{0}, \\
d_{L H}^{\prime 0}=\alpha_{d}^{0} \times d_{L H}^{0}, & d_{H H}^{\prime 0}=\alpha_{d}^{0} \times d_{H H}^{0} .
\end{aligned}
$$

The approximation subband, denoted $I^{\prime 1}$, is then reconstructed (see section II-C) and the number of levels is reduced to $N_{l}-1$. The $N_{l}$ entropies (associated to $3 N_{l}-2$ subbands) are calculated again to update the weights $\alpha_{a}, \alpha_{d}^{1}$ (equation (11) with $\left.N_{l}=N_{l}-1\right)$. After that, these weights are applied on the coefficients $I^{\prime 1}, d_{H L}^{1}, d_{H L}^{1}, d_{H L}^{1}$ to build $I^{\prime 2}$ as explained above. This process is iterated until $N_{l}=0$ to reconstruct the coarse tone mapped HDR image denoted $\widetilde{I}_{L D R}^{J}$, called coarse LDR image.

\section{Third stage: Reconstruction of the coarse LDR image}

The reconstruction stage is carried out inversely to the decomposition stage. Assume that the adaptive lifting scheme backward algorithm processed all resolution levels until $j-1$. The next step consists to recover the approximation coefficients $I^{\prime j}$ of size $N^{j} \times M^{j}$ using the four weighted blocks $I^{\prime j-1}, d_{L H}^{j-1}, d_{H L}^{\prime j-1}$ and $d_{H H}^{\prime j-1}$. The algorithm first deals with the coefficients in a vertical direction using $I^{j-1}$ and $d^{\prime j-1}$ (denoted $d^{\prime j-1}$ ). At a given $m$, the approximation coefficient at odd position, denoted $W^{\prime j}\left(x_{2 k-1}, y_{m}\right)$, is deduced:

$$
\begin{array}{r}
W^{\prime j}\left(x_{2 k-1}, y_{m}\right)=\hat{W}^{\prime j}\left(x_{2 k-1}, y_{m}\right)-d^{\prime j-1}\left(x_{k}, y_{m}\right) \\
\text { for } 1 \leq k \leq N^{j} / 2,
\end{array}
$$

where $\hat{W}^{\prime j}\left(x_{2 k-1}, y_{m}\right)$ is the predicted coefficient, at odd position, deduced from the weighted combination $v_{i}^{j-1}$ (with $i=0,1,2)$ of the neighboring approximation coefficients $I^{\prime j-1}\left(x_{2 k-1}, y_{m}\right)$ as:

$$
\begin{array}{r}
\hat{W}^{\prime j}\left(x_{2 k-1}, y_{m}\right)=\sum_{i=0}^{2} v_{i}^{j-1}\left(y_{m}\right) \cdot I^{\prime j-1}\left(x_{k+i-1}, y_{m}\right) \\
\text { for } 1 \leq k \leq N^{j} / 2 .
\end{array}
$$

The weights $v_{i}^{j-1}\left(y_{m}\right)$ are those computed and stored in the decomposition process. The approximation coefficient $W^{\prime j}\left(x_{2 k}, y_{m}\right)$, at even position, is deduced in a CA scheme:

$$
\begin{array}{r}
W^{\prime j}\left(x_{2 k}, y_{m}\right)=2 I^{\prime j-1}\left(x_{k}, y_{m}\right)-W^{\prime j}\left(x_{2 k-1}, y_{m}\right) \\
\text { for } 1 \leq k \leq N^{j} / 2 .
\end{array}
$$

The odd and even approximation coefficients are then merged to built 1D-signal:

$\left\{W^{\prime j}\left(x_{k}, y_{m}\right)\right.$ for $\left.1 \leq k \leq N^{j}\right\}$ $:=\left\{W^{\prime j-1}\left(x_{2 k-1}, y_{m}\right), W^{\prime j-1}\left(x_{2 k}, y_{m}\right)\right.$ for $\left.1 \leq k \leq N^{j} / 2\right\}$.

This process is repeated for all $m$ to reconstruct $W^{\prime j}$ of size $N^{j} \times M^{j} / 2$. These same steps are applied on $d_{H L}^{\prime j-1}$ and $d_{H H}^{\prime j-1}$ to generate the new block $U^{\prime j}$ of size $N^{j} \times M^{j} / 2$. As in the decomposition strategy, $W^{\prime j}$ and $U^{\prime j}$ are renamed $V^{\prime j-1}$ and $d^{\prime j-1}$. The same steps, as described above, are then performed but according to a horizontal direction. The reconstruction is iterated to finally build the image called coarse LDR image which is denoted $\widetilde{I}_{L D R}^{J}$.

\section{Fourth stage: Piecewise linear perceptual quantizer}

This stage proposes to adjust locally the distribution of the coarse LDR image logarithm luminance $\widetilde{I}_{L D R}^{J}$ according to the HVS to enhance the contrast using a piecewise linear function. This strategy is inspired from [19] which has been developed for compression purpose. However, modifications are made mainly to avoid the problem of empty bins of equal size.

To do so, the $\widetilde{I}_{L D R}^{J}$ values are first sorted and classified into equal $B$ bins defined by cutting points denoted $c_{u L D R}^{i}$ (with $1 \leq i \leq B$ ). A non-uniform histogram equalization is also performed. $c_{n u L D R}^{i}$ (with $1 \leq i \leq B$ ) cutting points, defining the bounds of the non-uniform consecutive $B$ bins, are deduced. The lower bound (cutting point) of each bin is then adjusted as follows:

$$
\widetilde{l}_{L D R}^{i}(1)=c_{u L D R}^{i}+\beta\left(c_{n u L D R}^{i}-c_{u L D R}^{i}\right),
$$

where $\beta$ is a positive parameter smaller than 1 .

Therefore the $\widetilde{I}_{L D R}^{J}$ values are classified into non-uniform $B$ bins as follows:

$$
\begin{array}{r}
\widetilde{I}_{L D R}^{J}=\left\{\widetilde{l}_{L D R}(k) \text { for } k=1, \ldots, N^{J} \times M^{J}\right\}= \\
\left\{\left[\widetilde{l}_{L D R}^{1}(1), \ldots, \widetilde{l}_{L D R}^{1}\left(K_{1}\right)\right], \ldots,\left[\widetilde{l}_{L D R}^{i}(1), \ldots, \widetilde{l}_{L D R}^{i}\left(K_{i}\right)\right], \ldots,\right. \\
\left.\left[\widetilde{l}_{L D R}^{B}(1), \ldots, \widetilde{l}_{L D R}^{B}\left(K_{B}\right)\right]\right\},
\end{array}
$$

depending on the quantization level set, where $K_{i}$ is the number of values in the $i-t h$ bin (i.e. $1 \leq i \leq B ; K_{i}>0$ ) and satisfying the following relation $\sum_{i=1}^{B} K_{i}=N^{J} \times M^{J}$.

The "s-shape" TM perceptual curve, as discussed in [4] and [5], is modelled by a piecewise linear curve on each bin (see Fig. 1). Consider the $i-t h$ bin, defined by $\left[\widetilde{l}_{L D R}^{i}(1), \ldots, \widetilde{l}_{L D R}^{i}\left(K_{i}\right)\right]$, the coarse LDR values are then modeled as follows:

$$
\hat{l}_{L D R}^{i}(k)=a^{i} \widetilde{l}_{L D R}^{i}(k)+b^{i} \text { with } k \in\left[1, K_{i}\right],
$$

where $a^{i}$ (with $a^{i} \neq 0$ ) and $b^{i}$ are two unknown parameters depending on the $i-t h$ bin. This equation, after some mathematical manipulations, can be rewritten as follows:

$$
\widetilde{l}_{L D R}^{i}(k)=\frac{\hat{l}_{L D R}^{i}(k)-\hat{l}_{L D R}^{i}(1)}{a^{i}}+\widetilde{l}_{L D R}^{i}(1),
$$

where the unknown parameter $a^{i}$ is deduced so that the MSE between the coarse LDR value and its quantized version, denoted $\bar{l}_{L D R}^{i}(k)$ (i.e. when $\hat{l}_{L D R}^{i}(k)$ has been supported a 
ceiling process according to a scalar quantization), is minimized in the $i-t h$ bin:

$$
\underset{a^{i}}{\arg \min }\left\|\bar{l}_{L D R}^{i}(k)-\widetilde{l}_{L D R}^{i}(k)\right\|_{2}^{2} .
$$

This equation is simplified as follows:

$$
\arg \min _{a^{i}} \sum_{k=1}^{K_{i}}\left(\frac{l_{L D R}^{i}(k)-\hat{l}_{L D R}^{i}(k)}{a^{i}}\right)^{2} \cdot p_{i},
$$

where $p_{i}$ is the probability of the $\widetilde{l}_{L D R}^{i}(k)$ value in the $i-t h$ bin given by $p_{i}=\frac{K_{i}}{\sum_{i=1}^{B} K_{i}}$.

Extending equation (22) to all bins involves the computation of a Global MSE (GMSE) deduced as follows:

$$
G M S E=\sum_{i=1}^{B} \sum_{k=1}^{K_{i}}\left(\frac{l_{L D R}^{i}(k)-\hat{l}_{L D R}^{i}(k)}{a^{i}}\right)^{2} \cdot p_{i} .
$$

The variance of $\left(l_{L D R}^{i}(k)-\hat{l}_{L D R}^{i}(k)\right)$ on each bin is assumed to be equal and is denoted $\xi$. Equation (23) is then simplified and becomes:

$$
G M S E=\sum_{i=1}^{B} \frac{p_{i}}{\left(a^{i}\right)^{2}} \cdot \xi
$$

Denote $l_{L D R \max }$ (respectively $l_{L D R \min }$ ) the maximum (respectively minimum) LDR luminance value. Introduce $\delta^{i}$ as the difference between coarse LDR luminance in two consecutive bins:

$$
\delta^{i}=\tilde{l}_{L D R}^{i+1}(1)-\tilde{l}_{L D R}^{i}(1) .
$$

A constraint related to the limit sum of the projected heights equal to the entire LDR range results in:

$$
\sum_{i=1}^{B} a^{i} \cdot \delta^{i}=l_{L D R \max }-l_{\text {LDRmin }} .
$$

Therefore the optimization problem is written as follows:

$\underset{a^{i}}{\arg \min } \sum_{i=1}^{B} \frac{p_{i}}{\left(a^{i}\right)^{2}} \cdot \xi, \quad$ s.t $\quad \sum_{i=1}^{B} a^{i} \cdot \delta^{i}=l_{L D R \max }-l_{L D R \min }$

This problem is solved analytically using the Lagrangian function. After some mathematical manipulations, the slope $a_{i}$ is deduced:

$$
a^{i}=\frac{\left(l_{L D R \max }-l_{L D R \min }\right) \cdot\left(p_{i}\right)^{1 / 3}}{\sum_{i=1}^{B} \delta^{i} \cdot\left(p_{i}\right)^{1 / 3}} .
$$

Hence the unknown parameter $b^{i}$ is calculated (i.e. $b^{i}=$ $\left.\hat{l}_{L D R}^{i}(1)-a^{i} \times \widetilde{l}_{L D R}^{i}(1)\right)$ and LDR mapped values are deduced according to equation (19). The global piecewise linear curve is continuous and strictly monotonic increasing according to the positive slopes (i.e $a^{i}>0$, or angles $0^{\circ}<\operatorname{atan}\left(a^{i}\right)<90^{\circ}$ ).

\section{Simulation RESUlts}

This section provides the performance of the proposed tone mapped HDR image. The tone mapped image quality is measured with the TMQI (Tone-Mapped image Quality Index) metric [18]. Simulations have been conducted under Matlab environnement using the HDR Toolbox ( [1]) with 274 test HDR images. For lack of space, we only present the results obtained with 8 HDR images ("Anturium", "Bottle Small", "Office", "Oxford Church", "Memorial", "Light",
"WardFlowers" and "StreetLamp") with different dynamic range (or contrast ratio) from $8 \mathrm{f}$-stops to $19 \mathrm{f}$-stops.

The proposed approach is compared to: (i) NONSEP ENO$\mathrm{CA}^{[13]}$; (ii) SEP ENO-CA ${ }^{[12]}$ with parameters $\alpha_{1}=0.3$, $\alpha_{2}=0.7$; (iii) Li TMO $^{[8]}$ with Haar multiscale; (iv) Fattal ${ }^{[11]}$ using RBW method with parameters $\alpha=0.8, \beta=0.3$, $\gamma=0.8$; (v) Duan ${ }^{[9]}$ using $\beta=0.5$; (vi) TMOs in HDR Toolbox: Drago ${ }^{[7]}$, Reinhard ${ }^{[14]}$, Ward ${ }^{[15]}$, Durand ${ }^{[6]}$, Schlick ${ }^{[17]}$ with the default parameters as given in the HDR Toolbox. The different parameters are chosen so as to give the best results in terms of TMQI metric in all methods.

Table I provides the TMQI metrics. The proposed TM approach namely "Proposed_LJ" is deployed with $B=256$, $\beta=0.25, l_{L D R \max }=255, l_{L D R \min }=0$ and $J=1, \ldots, 5$. Our approach is competitive to those developed in the literature. More the number of resolution levels increase, more the performance increase.

Fig. 2 compares the visual quality of the "Church" tone mapped image using "Duan" method and our approach. The stained glass window at the church background presents a better contrast and details with our approach although the TQMI are identical. Fig. 3 compares the "Memorial" tone mapped image using "Duan" and "Fattal" methods and our approach. The details on tills (see Fig. 3) and rosette (see Fig. 4) are better rendered by our approach.

Fig. 5 compares the visual quality of the "WardFlowers" tone mapped image using "Fattal" and our approach. Some details, on flowers and rocks, are lost on "Fattal" tone mapped image compared to our approach. Moreover, our tone mapped image if of better contrast. A similar result is provided by Fig. 6 where the HDR "StreetLamp" image has been mapped using "SEP_ENO" method and our method. The brightness of our tone mapped is better.

The performance of our approach is confirmed on more than 274 test HDR images where the details and contrast are better represented than other competitive methods.

\section{CONCLUSION}

This paper proposed a new HDR image TM approach able at the same time to extract the relevant details and enhance the contrast of LDR images. This is essentially related to : (i) the forward process of the near optimal local adaptive cell average lifting scheme where the filter coefficients are locally adapted to the content; (ii) the weighting operation depending on the information of each subband; (iii) the adjustment of the coarse LDR image luminance distribution according to the perceptual piecewise linear function. Simulation results confirm the relevance of the proposed approach both in terms of the TMQI metric and the visual quality of the displayed image. 


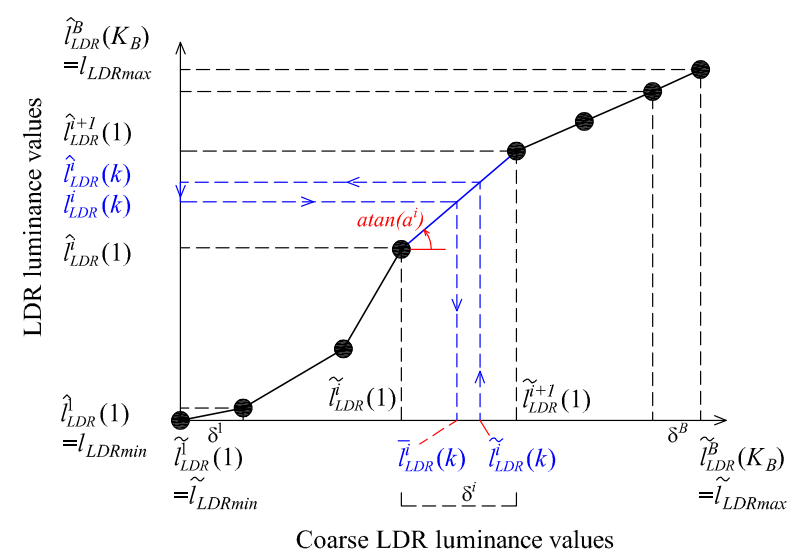

Fig. 1: Piecewise linear curve modelization ("s-shape" curve).
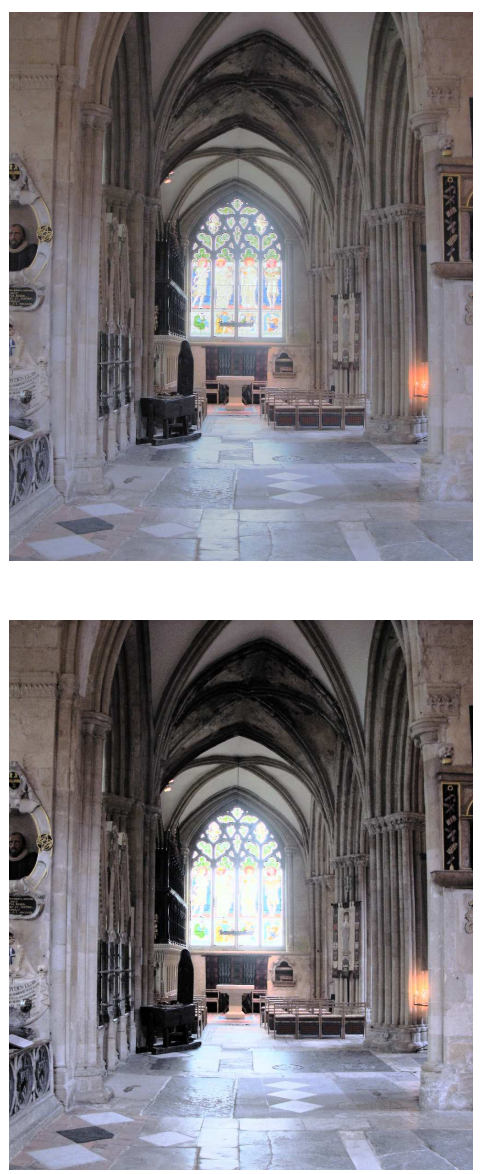

Fig. 2: "Oxford Church" HDR test image (15.46 f-stops) Up image: proposed (5 levels, TMQI $=0.985)$; Down image: "Duan" (TMQI=0.986).
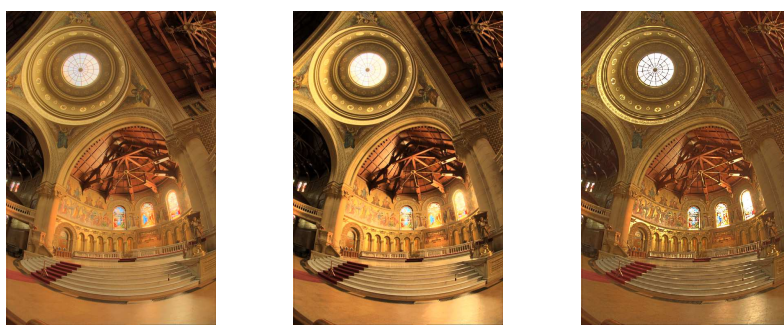

Fig. 3: "Memorial" HDR test image (18.38 f-stops) - Left image: proposed (5 levels, TMQI=0.951); Middle image: "Duan" (TMQI=0.935); Right image: "Fattal" (TMQI=0.927).
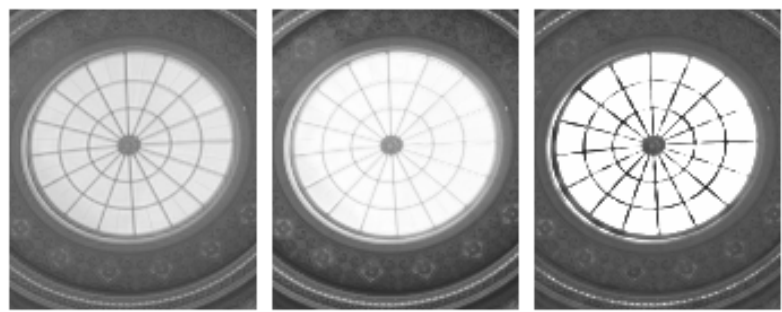

Fig. 4: LDR luminance "Rosette" zoom - Left image: proposed (5 levels, TMQI=0.951); Middle image: "Duan" (TMQI=0.935); Right image: "Fattal" (TMQI=0.927).
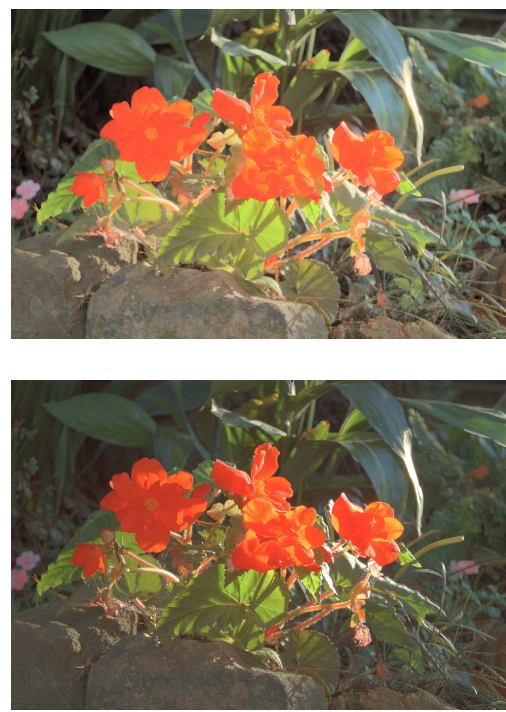

Fig. 5: "WardFlowers" HDR test image (14.01 f-stops) - Up image: proposed (5 levels, TMQI $=0.930)$; Down image: "Fattal" (TMQI=0.875). 


\section{REFERENCES}
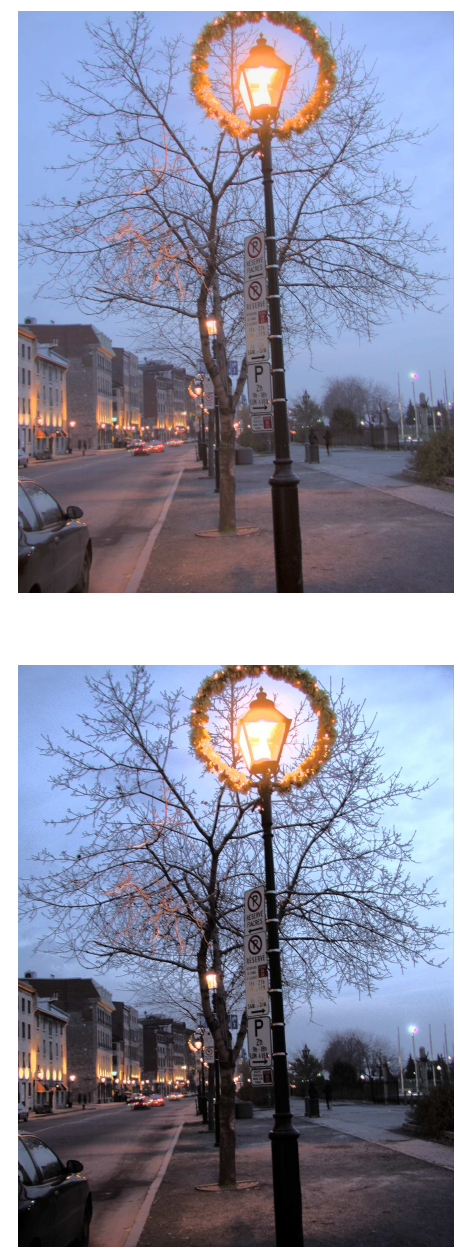

Fig. 6: "StreetLamp" HDR test image (13.83 f-stops) - Up image: proposed (5 levels, TMQI=0.911); Down image: "SEP_ENO" (TMQI=0.855).

TABLE I: Tone Mapped Image Quality Index (TMQI)

\begin{tabular}{lclllll}
\hline TMOs & Anturium & Bottle & Office & Church & Memorial & Light \\
DR f-stops & 8.73 & 16.03 & 16.29 & 15.46 & 18.38 & 17.46 \\
\hline Drago $^{[7]}$ & 0.874 & 0.801 & 0.800 & 0.814 & 0.800 & 0.800 \\
Reinhard $^{[14]}$ & 0.778 & 0.807 & 0.826 & 0.789 & 0.791 & 0.794 \\
Ward $^{[15]}$ & 0.806 & 0.783 & 0.775 & 0.817 & 0.795 & 0.789 \\
Durand $^{[6]}$ & 0.811 & 0.892 & 0.825 & 0.929 & 0.814 & 0.800 \\
Tumblin $^{[16]}$ & 0.715 & 0.713 & 0.735 & 0.675 & 0.759 & 0.750 \\
Schlick $^{[17]}$ & 0.770 & 0.835 & 0.926 & 0.970 & 0.787 & 0.780 \\
Duan $^{[9]}$ & $\mathbf{0 . 9 6 4}$ & 0.916 & $\mathbf{0 . 9 5 5}$ & $\mathbf{0 . 9 8 6}$ & $\mathbf{0 . 9 3 5}$ & 0.969 \\
Fattal $^{[11]}$ & 0.889 & 0.928 & 0.943 & 0.889 & 0.927 & $\mathbf{0 . 9 7 1}$ \\
Li $^{[8]}$ & 0.964 & $\mathbf{0 . 9 5 4}$ & 0.854 & 0.877 & 0.834 & 0.888 \\
SEP_ENO $[12]$ & 0.896 & 0.934 & 0.943 & 0.895 & 0.932 & 0.970 \\
NONSEP ${ }^{[13]}$ & 0.938 & 0.873 & 0.935 & 0.820 & 0.832 & 0.932 \\
\hline Proposed_L1 & 0.946 & 0.863 & 0.934 & 0.954 & 0.918 & 0.954 \\
Proposed_L2 & 0.965 & 0.882 & 0.938 & 0.970 & 0.929 & 0.963 \\
Proposed_L3 & 0.978 & 0.904 & 0.945 & 0.981 & 0.941 & 0.966 \\
Proposed_L4 & 0.980 & 0.921 & 0.946 & 0.984 & 0.949 & 0.969 \\
Proposed_L5 & $\mathbf{0 . 9 8 2}$ & $\mathbf{0 . 9 3 3}$ & $\mathbf{0 . 9 4 8}$ & $\mathbf{0 . 9 8 5}$ & $\mathbf{0 . 9 5 1}$ & $\mathbf{0 . 9 6 9}$ \\
\hline
\end{tabular}

[1] Banterle, F., Artusi, A., Debattista, K., and Chalmers, A., Advanced High Dynamic Range Imaging: Theory and Practice, AK Peters (now CRC Press), ISBN: 978-156881-719-4 (2011).

[2] Dufaux, F., Le Callet, P., Mantiuk, R., Mrak, M., High Dynamic Range Video 1st Edition : From Acquisition, to Display and Applications, ISBN: 9780081004128 (April 2016)

[3] Reinhard, E., Heidrich, Wolfgang., Debevec, Paul., Pattanaik, S., Ward, G., and Myszkowski, K., High Dynamic Range Imaging 2nd Edition: Acquisition, Display, and Image-Based Lighting, ISBN: 9780123749147 (May 2010).

[4] Dowling, J. E., The Retina: An Approachable Part of the Brain, Cambridge, Belknap Press (1987).

[5] Geisler, W. S., Effects of Bleaching and Backgrounds on the Flash Response of the Cone System, Journal of Physiology, 312:413-434 (1981).

6] Durand, F., and Dorsey, J., Fast Bilateral Filtering for The Display of High-dynamic-range Images, ACM Transactions on Graphics (TOG) Proceedings of ACM SIGGRAPH 21, pp. 257-266 (2002).

[7] Drago, F., Myszkowski, K., Annen, T., and Chiba, N., Adaptive Logarithmic Mapping for Displaying High Contrast Scenes, Computer Graphics Forum 22, pp. 419-426 (2003).

[8] Li, Y., Sharan, L., and Adelson, E.H., Compressing and Companding High Dynamic Range Images with Subband Architectures, ACM Trans. Graph. 24, pp. 836-844 (July 2005).

[9] Duan, J., Bressan, M., Dance, C., and Qiu, G., Tone-mapping High Dynamic Range Images by Novel Histogram Adjustment, Pattern Recognition, vol. 43, pp. 1847-1862 (2010).

[10] Husseis, A., Mokraoui, A., and Matei, B., Revisited Histogram Equalization as HDR Images Tone Mapping Operators, 17th IEEE International Symposium on Signal Processing and Information Technology, ISSPIT (December 2017).

[11] Fattal, R., Edge-Avoiding Wavelets and their Applications, ACM Trans Graph (2009).

[12] Thai, B.C., Mokraoui, A., and Matei, B., Performance Evaluation of High Dynamic Range Image Tone Mapping Operators Based on Separable Non-linear Multiresolution Families, 24th European Signal Processing Conference, pp. 1891-1895 (August 2016).

[13] Thai, B.C., Mokraoui, A., and Matei, B., Image Tone Mapping Approach Using Essentially Non-Oscillatory Bi-quadratic Interpolations Combined with a Weighting Coefficients Strategy, 17th IEEE International Symposium on Signal Processing and Information Technology, ISSPIT (December 2017).

[14] Reinhard, E., and Devlin, K., Dynamic Range Reduction Inspired by Photoreceptor Physiology, IEEE Transactions on Visualization and Computer Graphics 11, pp. 13-24 (2005).

[15] Ward, G., Rushmeier, H., and Piatko, C., A Visibility Matching Tone Reproduction Operator for High Dynamic Range Scenes, IEEE Transactions on Visualization and Computer Graphics 3, pp. 291-306 (1997).

[16] Tumblin, J., and Rushmeier, H., Tone Reproduction for Realistic Images IEEE Comput. Graph. Appl, pp. $42-48$ (1993).

[17] Schlick, C., Quantization Techniques for Visualization of High Dynamic Range Pictures, In Proceeding of the Fifth Eurographics Workshop on Rendering, pp. 7-18 (1994).

[18] Yeganeh, H. and Wang, Z., Objective Quality Assessment of Tonemapped Images, IEEE Trans. on Image Processing, vol. 22, pp. 657-667 (February 2013)

[19] Mai, Z., Mansour, H., Nasiopoulos, P., and Ward, R., Visually-favorable tone-mapping with high compression performance, Image Processing (ICIP) 17th IEEE International Conference on, pp. 1285-1288, ISSN 1522-4880 (2010) 